\title{
A simple device to inject indicator gas for wash-out tests during mechanical ventilation
}

\author{
I. Gültuna, P.E.M. Huygen, C. Jabaaij, W. P. J. Holland, C. Ince and H. A. Bruining \\ Intensive Care Unit, Department of Surgery, University Hospital of Rotterdam, Rotterdam, The Netherlands
}

Received: 17 August 1991; accepted: 25 May 1992

\begin{abstract}
Objective: To evaluate a simple device which injects a constant fraction of indicator gas to the inspiratory mixture for performing multi-breath wash-out tests during controlled ventilation. Design: the technique in which the indicator gas is injected at the mouth of the patient (post-mix) is compared with the technique where the indicator gas is administered in the bellows of the ventilator (pre-mix). Setting: Surgical Intensive Care Unit of a University Hospital. Patients: 10 post-operative mechanically ventilated patients. Interventions: None. Measurements and results: 3 wash-out tests with the post-mix and 3 wash-out tests with the pre-mix method were performed within an hour on every patient. The calculated mean end expiratory lung volume (EEV) was $1.91 \pm 0.871$ with the post-mix technique and $1.89 \pm 0.881$ with the premix technique. There was a good agreement with a mean difference of $-1.9 \pm 6.5 \%$ in the calculated EEV values by the two different techniques. Conclusion: The described injector is an affordable device, is easy to assemble and can be incorporated in most electronically regulated ventilators to perform multi-breath indicator gas wash-out tests for pulmonary monitoring at the bed side of ICU patients.
\end{abstract}

Key words: Indicator gas - Wash-out test - Critical orifice end expiratory volume - Mechanical ventilation

A multi-breath indicator gas wash-out test provides a non-invasive way of monitoring the lungs of mechanically ventilated patients at the bedside in an intensive care unit (ICU). This test can be used to identify both restrictive and obstructive lung diseases $[1,2]$. There are already several methods described to perform wash-out tests. In most described wash-out techniques [1-4] a perfect stepwise change of the indicator gas fraction is not achieved due to gas remaining in the bellows and tubing of the ventilator at the beginning of a wash-out. To compensate for this, complex corrections are usually implemented [5]. Richardson et al. [6] described an automated $\mathrm{N}_{2}$ washout method where a slider valve was used to achieve a step-wise change in the $\mathrm{N}_{2}$ fraction. However the often performed $\mathrm{N}_{2}$ wash-out tests cause large variations in the $\mathrm{FiO}_{2}$ which interfere with the care of the patient and are therefore not suitable for use in critically ill patients. In wash-out tests where no step-wise change of indicator gas is achieved, information about ventilation inhomogeneity is more difficult to extract from the data [2].

We previously described a device for injecting indicator gases for wash-out tests in mechanically ventilated patients [1] where the indicator gas is mixed with the ventilatory gases prior to entering the ventilator (pre-mix). This method can only be used with a low pressure gas inlet ventilator and cannot achieve a perfect step-wise change in the indicator gas fraction during the initial breaths of a wash-out at the mouth of the patient.

In the last few years new techniques have been described in which a step-wise change of small fractions of indicator gas is achieved by injecting the indicator gas at the mouth. East et al. [7, 8] and Larsson et al. [9] used complex systems to perform wash-out tests with constant indicator gas fractions, suitable for most types of ventilatory modes. Jonmarker et al. $[10,11]$ described a technique suitable for a controlled ventilation mode. However, it is uncertain whether their method indeed gave a constant inspiratory indicator gas fraction as this was not measured during the wash-in.

This paper describes the design of a simple device which can be used with most electronically regulated ventilators as an indicator gas injector in a controlled ventilatory mode. The performance of the injector method was compared with the pre-mix method in mechanically ventilated ICU patients.

\section{Material and methods}

\section{Design of the injector}

The injector device is designed to inject a constant flow of indicator gas into a constant flow of air/oxygen mixture delivered by the ventilator during the inspiration. The constant injection flow is achieved with a critical orifice (Euroglas, Delft, The Netherlands). The critical orifice is a small diverging opening in a glass capillary which generates a constant 


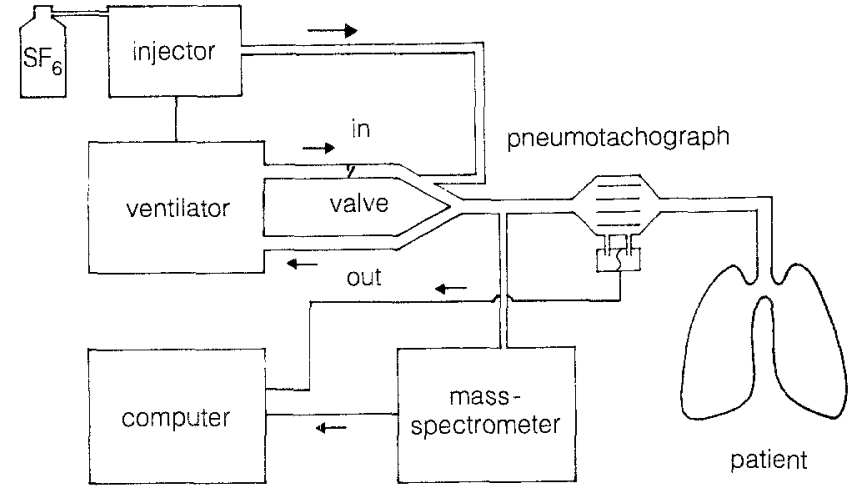

Fig. 1. The measurement set-up. The indicator gas is injected before the Y-piece at the end of the inspiratory tubing. During inspiration a constant flow of indicator gas is supplied by a critical orifice. The respiratory flow is measured by a pneumotachograph and the indicator gas is sampled and measured by a mass-spectrometer. The end expiratory lung volume is automatically calculated by the computer

flow when the absolute pressure upstream of the critical orifice is at least two times the downstream pressure. The flow is proportional to the upstream pressure and is independent of the downstream pressure. The final design of the injector is shown in Fig. $2 \mathrm{~d}$, where a two-way valve (Kuhnke, $24 \mathrm{~V} \mathrm{DC}$, Germany) is placed upstream and a three-way valve downstream of the critical orifice. The reason for this double valve construction will be mentioned in the results. The valves are triggered by the ventilator and are opened synchroneously at the onset of an inspiration and closed at the end of an inspiration. The indicator gas is injected countercurrent to the flow just before the Y-piece at the end of the inspiratory tube. A one-way valve placed proximal to the injection orifice prevents diffusion of the indicator gas into the inspiratory tubing (Fig. 1). The fraction of the indicator gas during injection to the patient is determined by the ratio of the inspiratory flow delivered by the ventilator to the injector flow. The critical orifice used generates an indicator gas flow of approximately $500 \mathrm{ml} / \mathrm{min}$ with an effective upstream pressure of 2 bar. This means that when a patient is ventilated with a minute volume of $91 / \mathrm{min}$ and an inspiration time ratio of $25 \%$, the indicator gas fraction administered will be $1.4 \%$. A programmable time relay (CPX-519, 24 V DC, Comat AG, Switzerland) allows the indicator gas fraction to be delivered after a delay following the onset of an inspiration and is adjustable in steps of $0.01 \mathrm{~s}$. With a time delay adjustment, the indicator gas flow can be synchronized with the ventilator gas flow pattern as will be described in the results.

\section{Measurement set-up}

The injector is connected to a Servo 900-B ventilator (Siemens Elema, Sweden) where the indicator gas is injected just before the Y-piece at the end of the inspiratory tubing as shown in Fig. 1. The injection is not between the Y-piece and the mouth, because at the beginning of the inspiration this limb will be filled with the end expiratory gas, that will still contain indicator gas. As this residual indicator gas combines with the injected constant indicator gas fraction, a higher indicator gas fraction in the beginning of every inspiration will occur during a wash-in. The indicator gas used in this study is Sulphur Hexafluoride $\left(\mathrm{SF}_{6}\right)$ which is an inert and poorly soluble gas. The $\mathrm{SF}_{6}$ gas tube is connected to the injector through a two stage pressure regulator (Messer) Griesheim, Germany) which keeps the input pressure constant. Downstream from the point of injection in the ventilator tubing, the indicator and metabolic gases are continuously sampled by a mass-spectrometer (Airspec, MGA 3000, Chest Instruments, Biggin Hill, U.K.) with a teflon capillary. The rise time of the mass-spectrometer is $40 \mathrm{~ms}$ and the sampling occurs at a rate of $200 \mathrm{ml} / \mathrm{min}$, causing a $330 \mathrm{~ms}$ delay, which is adjusted for by a signal processing algorithm. The respiratory flow is measured with a pneumotachograph (Fleisch-2, Gould-Godard, The Netherlands), connected to a differential pressure transducer (Validyne MP 45-14-871, 0-2 mBar, Northridge, CA, USA), placed near the mouth. All the data are sampled by a computer (PDP-11, DEC, Maynard, MA) at $100 \mathrm{~Hz}$ and the EEV is calculated using the algorithm of Zwart [12] and Huygen [1, 13], based on the relation between the dilution of the indicator gas in the lung and the net amount of indicator gas washed out.

\section{Measurements and the patient group}

In the course of the development of this injector, different configurations with the valves were tested on a dummy lung while the inspiratory indicator gas fractions were analysed.

The new injector was evaluated in a bench test, by comparison of the calculated EEV values obtained from wash-out tests performed with the post-mix injector method to that obtained by the pre-mix method [1]. These measurements were performed on a dummy lung (Dräger, Lübeck, Germany) with a volume of $3.05 \pm 0.01 \mathrm{~L}$ (volume determined using a Helium dilution technique).

The injector was clinically validated on 10 post-operative mechanically ventilated patients with a mean age of 68 years (range 54-81). Of these patients 3 had a normal lung function and 7 suffered from COPD of which 2 had emphysema. Measurements on patients were commenced as soon as the patients arrived at the ICU. All patients, except one multitrauma patient, had undergone an upper abdominal operation (elective aortic or oesophageal surgery) and consented to the application of the wash-out tests pre-operatively. All patients were pulmonary as well as hemodynamically stable during the wash-out tests and all the COPD patients were optimally pre-medicated. Patients were ventilated in the IPPV mode except the trauma patient who had $15 \mathrm{~cm} \mathrm{H}_{2} \mathrm{O}$ of PEEP because of multiple rib fractures and a bilateral lung contusion. Since the patients were sedated the tidal volumes remained constant and did not affect the EEV values during the wash-out test period.

Prior to a wash-out test being initiated, the indicator gas was washed in for at least 5 minutes until the difference between the inspiratory and expiratory indicator gas fraction was less than $2 \%$ of the inspiratory fraction. After the indicator gas had been washed in, a wash-out test was started by cessation of the injection of the indicator gas. Six washout tests, each performed for $5 \mathrm{~min}, 3$ wash-outs using the pre-mix method and 3 wash-outs using the injector method were performed on every patient and measurements were completed within an hour. From these wash-out tests the EEV values were calculated and compared with each other.

\section{Results}

In the development of the injector we analysed the inspiratory indicator gas fractions. This revealed the presence of an initial spike found to be caused by two different factors: 1) The transience between the onset of an inspiration at the ventilator and the injection of the indicator gas flow, that can be corrected by the application of a delay in the injector flow. 2) The build up of a high pressure in the injector when only a two-way valve is used.

The initial spike caused by the asynchronicity between the indicator and ventilator gas flow is negligible compared to the initial spikes caused by the pressure build up in the injector when a simple two-way valve is used. A $60 \mathrm{~ms}$ time delay is used to eliminate the effect of asynchronicity of the ventilator and the indicator gas flow when the maximum inspiratory flow is limited to $60 \mathrm{l} / \mathrm{min}$. In Fig. $2 \mathrm{a}$, when only a two-way valve is placed proximal to the critical orifice, the lumen between the valve and critical orifice will contain gas with the high inlet pressure when the valve closes at the end of the inspiration. During the next expiration this gas expands and flows into the non-moving gas mixture in the inspiratory 
tubing causing a bolus of undiluted indicator gas. This leads to an initial spike up to 16 times greater than the mean indicator gas fraction at the beginning of the next inspiration (Fig. $3 a$ ). Figure $2 b$ shows the effect of a twoway valve placed distal to the critical orifice. Here, pressure builds up in the tubing between the critical orifice and the valve when the valve is in the closed position during the expiration cycle. When the valve opens at the beginning of the inspiration, this pressurized gas in the tubing suddenly expands, and causes a high injection flow of short duration. This is reflected by the occurrence of an initial spike up to 21 times greater than the mean indicator gas fraction at the same inspiration (Fig. 3b). Figure $2 \mathrm{c}$ shows the option when a three-way valve is placed distal to the critical orifice. Here no spike will be formed as the pressure build up between the critical orifice and the valve is prevented by flushing, since the three-way valve opens to the atmosphere during the expiration cycle. A continuous flow of indicator gas during expiration is the drawback of this option. To prevent waste of indicator gas during the expiration, a two-way valve was placed before the critical orifice (Fig. 2 d). This became the final configuration of the injector device used clinically.

We observed an initial spike which is two times greater than the mean indicator gas fraction during the injection with the final configuration of the injector device, with

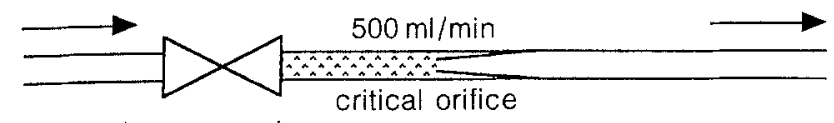

a two-way valve
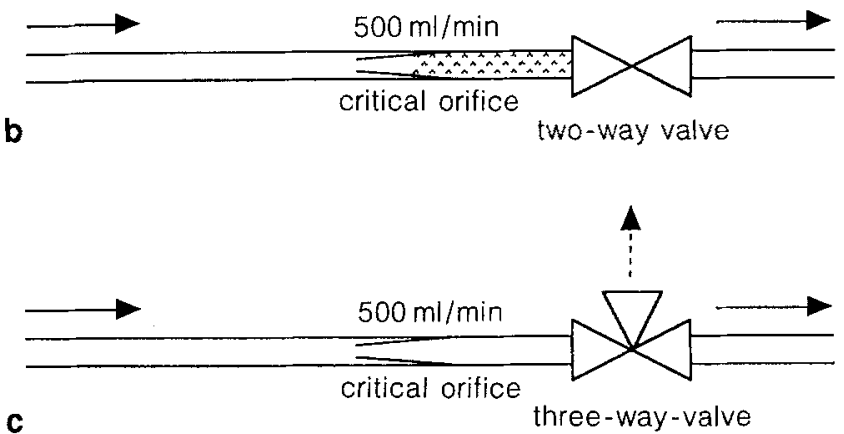

C

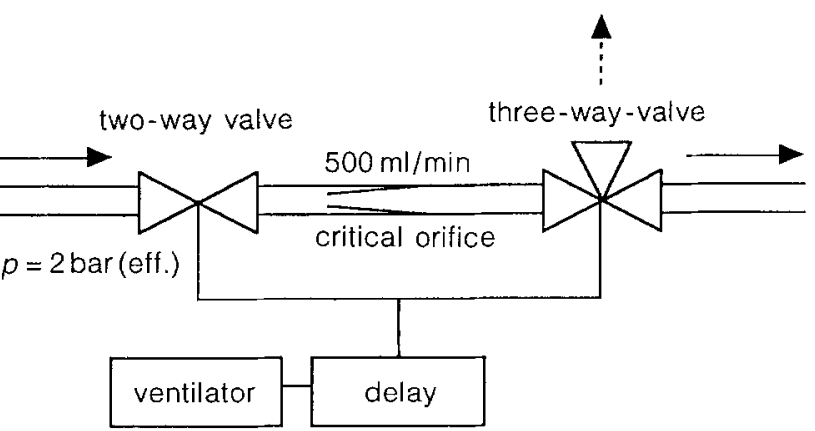

Fig. 2a-d. Schematic models of four options for the indicator gas injector are shown. In (a) a two-way valve is placed before the critical orifice whereas in (b) the two-way valve is placed after the critical orifice. In (c) a three-way valve is placed after the critical orifice and in (d) a two-way valve and a three-way valve are placed before and after the critical orifice respectively no time delay adjustment between the onset of the inspiration at the ventilator and the injection of the indicator gas (Fig. 4b). This initial spike is caused by the difference between the ventilator and the indicator gas flow at the beginning of an inspiration. In all 10 patients measured (with a minute ventilation range of $7-151$ and a maximum inspiratory flow range of $467-1000 \mathrm{ml} / \mathrm{s}$ ) a time delay of $60 \mathrm{~ms}$ gave the best step-wise change of the inspiratory indicator gas fraction and eliminated the observed initial spike during the injection (Fig. 4c). Figure 4 a shows the indicator gas fraction during a wash-in with the pre-mix method, where the indicator gas is injected before it enters the ventilator.

The mean volume calculated for the dummy lung from 5 wash-out tests using the injector method with a $60 \mathrm{~ms}$ time delay between the indicator gas injection and the onset of the inspiration, was $3.02 \pm 0.031$ which agreed well with the actual volume of $3.05 \pm 0.011$ (volume determined using a Helium dilution method).
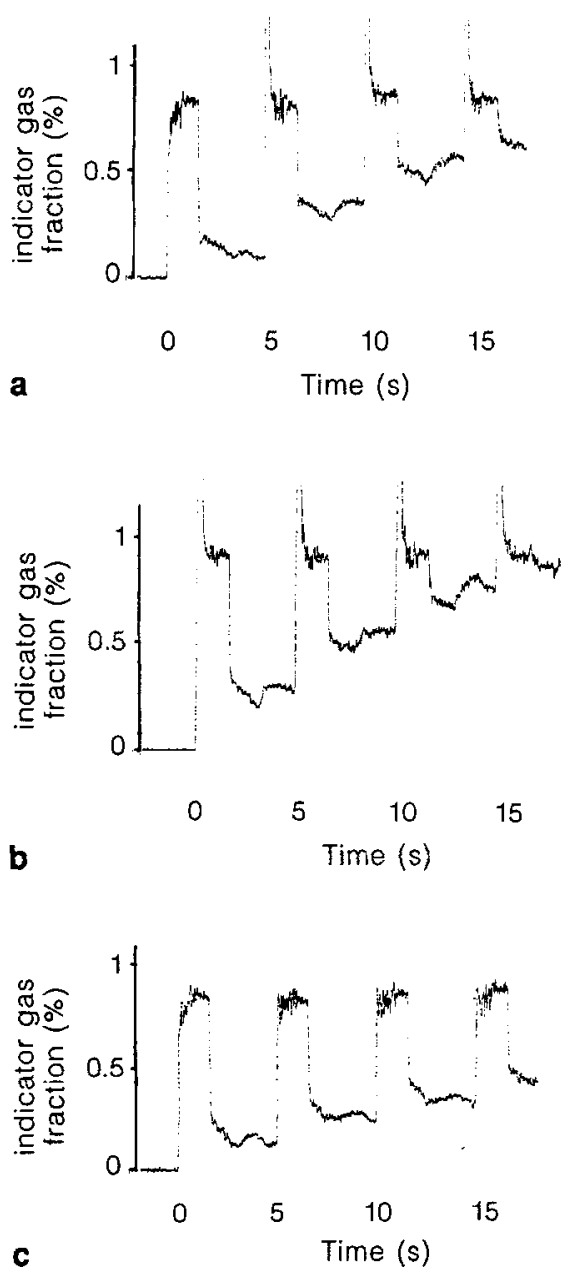

Fig. 3a-c. The indicator gas fractions during a wash-in procedure on a dummy-lung in each design option are shown with the application of a $60 \mathrm{~ms}$ time delay. If only a two-way valve is placed proximal to the critical orifice (Fig. 2 a) an initial spike will occur in the next inspiration up to 16 times greater than the mean indicator gas fraction (a). If a twoway valve is placed distal to the critical orifice (Fig. 2b) an initial spike up to 21 times greater than the mean indicator gas will occur in the same inspiration (b). In a combination of a two- and a three-way valve configuration (Fig. 2d) no spike will occur as pressure build-up is prevented (c) 

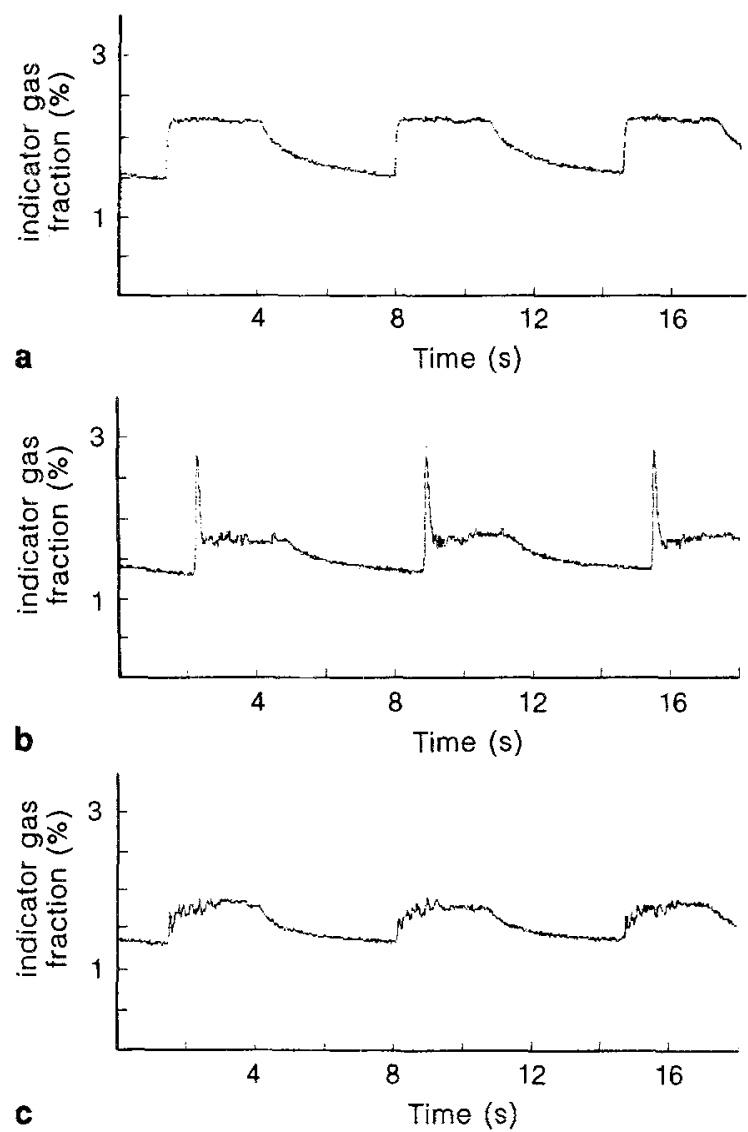

Fig. $4 a-c$. The indicator gas fraction during a wash-in procedure on a patient, with the pre-mix method (a), with the described injector device without a time delay, note the occurrence of an initial spike which is two times greater than the mean indicator gas fraction (b), and with the described injector device with the introduction of a $60 \mathrm{~ms}$ time delay between the start of the inspiration at the ventilator and the indicator gas injection, eliminates the initial spike (c)

The mean values of the calculated EEV measurements of the 10 patients are listed in Table 1 . The mean EEV values for all patients was $1.91 \pm 0.871$ (range $1.01-3.75 \mathrm{l})$ with the post-mix injector method and $1.89 \pm 0.881$ (range $0.89-3.61 \mathrm{l}$ ) with the pre-mix method. The intraindividual standard deviation for multiple mea- surements was $0.04 \pm 0.021$ with the post-mix injector and $0.04 \pm 0.031$ with the pre-mix method. The mean difference in the EEV values measured with both methods was $-1.9 \pm 6.5 \%$ (range $-13.5 \%$ to $+8.6 \%$ ). There was a good agreement between the calculated EEV values by the two different methods, all individual differences ranging within two times the standard deviation of the mean difference.

\section{Discussion}

There are not many tests available in the ICU setting to measure lung volumes at the bedside. A multi-breath indicator gas wash-out test provides a good method to identify obstructive and restrictive disorders but can not be routinely implemented at the bed-side because bulky and expensive devices are needed. An optimal injector method should accommodate the following; it should 1) be simple, 2) be capable to perform wash-out tests in all ventilatory modes, 3 ) be suitable for all kinds of ventilators, 4) be able to attain a constant inspiratory indicator gas fraction, 5) give a step-wise change in the indicator gas fraction, 6) be able to perform wash-out tests with small fractions of indicator gas.

The injector device described allows all options, except the capability to perform wash-out tests in all ventilatory modes. This is not an essential disadvantage, since most pulmonary deteriorated ICU patients are already ventilated in a controlled mode. We preferred to design a simple and affordable injector device. East [7] and Larsson [9] chose for the second option, making their setup more complex but able to perform wash-out tests in most ventilatory modes. East [7], Larsson [9] and Jonmarker [11] used a method based on similar principles to the device described in this paper. However, all these teams of authors injected the indicator gas $\left(\mathrm{SF}_{6}\right)$ distal to the Y-piece which could interfere with the indicator gas fraction. Jonmarker [11] injected the indicator gas between the mouth and the Y-piece where indicator gas will still be present in the tubing at the end of the expiration. The injection of a constant fraction of indicator gas during the inspiration causes higher fractions of indicator

Table 1. The results of the calculated end expiratory lung volumes (EEV) by the pre-mix (PM) and the injector method (I) in 10 mechanically ventilated patients

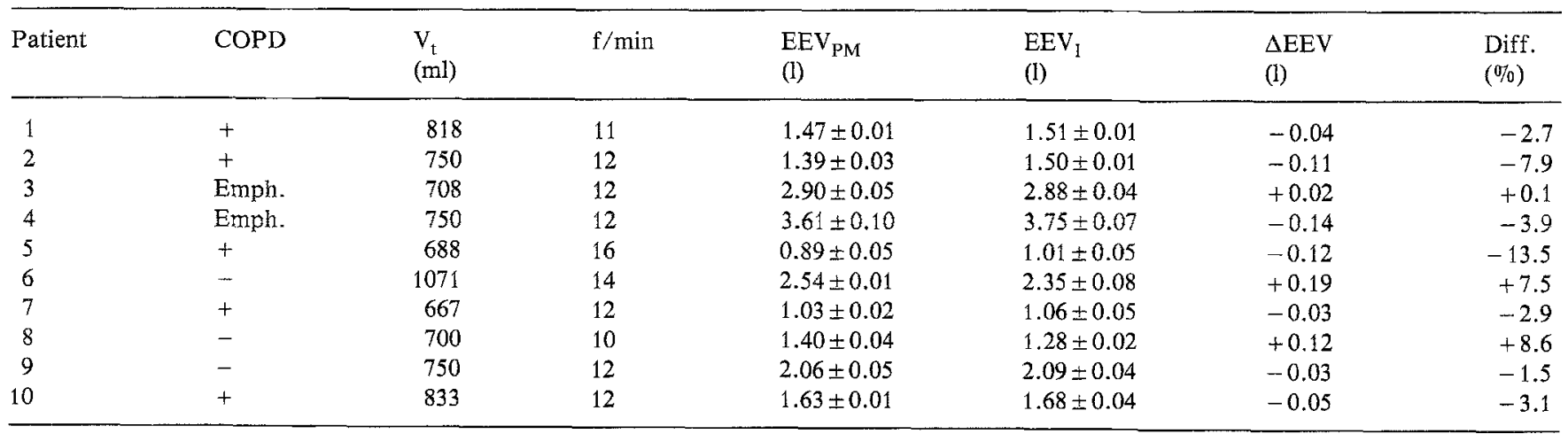

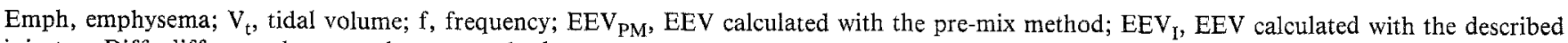
injector; Diff, difference between the two methods 
gas at the beginning of the inspiration due to residual indicator gas in the tubing at the beginning of the inspiration being added to the injected indicator gas. Larsson [9] avoided this by starting the injection with a delay, while East [7] corrected this by subtracting the rebreathed indicator gas from the injected indicator gas. Analysis of the inspiratory fractions in the present study revealed the presence of an initial spike caused by two different factors: a pressure build-up in the injector when only a twoway valve is used and a time delay between the onset of an inspiration at the ventilator and the injection of the indicator gas. These spikes at the beginning of the inspiration may result in an inhomogeneous mixing of the indicator gas in the lung possibly affecting the EEV calculations. A configuration of an injector with a two- and three-way valve in series and the application of an optimal time delay between the start of an inspiration at the ventilator and the indicator gas injection, eliminated the initial spikes and attained a constant indicator gas fraction during the injection. This was confirmed by the measurement of the inspiratory indicator gas fraction just after the injection orifice (Fig. $3 \mathrm{c}$ ). A limitation common to both our injector method and that of Jonmarker [11] is that these techniques can only be applied during IPPV and PEEP ventilation, as the inspiratory gas flow has to be constant. If necessary, the previously described premix method [1] can be used to perform wash-out tests in the IMV mode [14]. In other ventilatory settings or spontaneous ventilation where the inspiratory flow is not constant, the method of East [7] or Larsson [9] can be applied. Their technique is based on a more complex, feedforward mechanism in which the indicator gas flow to be injected is regulated by the inspiratory flow of the patient.

The post-mix injector method can be applied during PEEP ventilation. In our ICU this has proven to be very useful in the titration of the PEEP level for achieving a desired end expiratory lung volume in ARDS patients, avoiding the use of undesirable high levels of PEEP [15]. The information provided by calculation of EEV is not only relevant to restrictive lung disorders. This is shown in Table 1, where EEV measurements in two emphysema patients even under sedation and in supine position identified hyperinflation.

The indicator gas injector described in this study is a simple device, easily applied to most electronically regulated ventilators with low as well as high pressure inlets. As it is an affordable device it can be built into each ventilator present in the ICU, so that no disconnection is needed to commence a wash-out test during mechanical ventilation. Furthermore the original ventilator settings and the oxygen supply do not have to be altered by the measurements, since only small fractions of indicator gas are used. From the wash-out curves, not only the EEV can be calculated but additional information about the ventilation inhomogeneity, the deadspace ventilation and the ventilation-perfusion inhomogeneity can be obtained which can help in diagnosis and therapy $[12,15]$. In conclusion the device described in this paper allows continuous bedside pulmonary parameters to be measured and enables a non-invasive evaluation of the effects of drugs and ventilatory settings on the lungs of critically ill mechanically ventilated patients.

Acknowledgements. This study was in part supported by the Netherlands Asthma Foundation (Grant no. 84.12).

\section{References}

1. Huygen PEM, Feenstra BWA, Holland WPJ, Ince C, Stam H, Bruining HA (1990) Design and validation of an indicator gas injector for multiple gas wash-out tests in mechanically ventilated patients. Crit Care Med 18:754-759

2. Huygen PEM, Strijdhorst H, Ince C, Zwart A, Feenstra BWA, Bruining HA (1989) A new measure for pulmonary ventilation inhomogeneity using indicator gas washout test. Pflügers Arch 414:S198 (abstract)

3. Hylkema BS, Barkmeijer-Degenhart P, van der Mark TW (1982) Measurement of functional residual capacity during mechanical ventilation for acute respiratory failure. Chest $81: 27-30$

4. Paloski WH, Newell JC, Gisser DG (1981) A system to measure functional residual capacity in critically ill patients. Crit Care Med 9:342-346

5. Felton CR, Saidel GM, Montenegro HD (1984) Moment analysis of multibreath nitrogen washout with a variable input gas composition. Med Biol Eng Comput 22:486 - 492

6. Richardson P, Anderson M (1982) Automated nitrogen-washout methods for infants: evaluated using cats and a mechanical lung. $J$ Appl Physiol 52:1378-1382

7. East TD, Wortelboer PJM, van Ark E, Bloem FH, Lisheng Peng M, Pace NL, Crapo RO, Drews D, Clemmer TP (1990) Automated sulfur hexafluoride washout functional residual capacity measurement system for any mode of mechanical ventilation as well as spontaneous ventilation. Crit Care Med 18:84-91

8. East TD, Andriano KP, Pace NL (1987) Automated measurement of functional residual capacity by sulfur hexafluoride washout. $J$ Clin Monit 3:14-21

9. Larsson A, Linarsson D, Jonmarker C, Jonson H, Werner O (1987) Measurement of lung volume by sulphur hexafluoride wash-out during spontaneous and controlled ventilation. Further development of a method. Anesthesiology 67:543-550

10. Jonmarker C, Castor R, Drefeldt B, Werner O (1985) An analyser for in-line measurement of expiratory Sulphur hexafluoride concentration. Anesthesiology 63:84-88

11. Jonmarker C, Jansson L, Jonson B, Larsson A, Werner O (1985) Measurement of functional residual capacity by sulphur hexa fluoride washout. Anesthesiology 63:89-95

12. Zwart A (1988) Ventilation and perfusion distributions in lungs obtained by massspectrometry. In: Proceedings of the IEEE Engineering in Medicine Biology Society $10^{\text {th }}$ Annual International Conference, pp 820-822

13. Huygen PEM, Feenstra BWA, Hoorn E, Jansen JRC, Zwart A (1991) PDPS: a pulmonary data processing system for assessment of gas exchange properties by multiple gas wash-out. Comput Meth Progr Biomed 36:223-235

14. Bruining HA (1984) Two simple assemblies for the application of intermittent mandatory ventilation with positive end-expiratory pressure. Intensive Care Med 10:33-35

15. Gültuna I, Huygen PEM, Strijdhorst $H$, Ince C, Bruining HA (1990) Clinical applications of an indicator washout test during artificial ventilation. Intensive Care Med 16:165 (abstract)

I. Gültuna, MD

Department of Surgery

University Hospital of Rotterdam

Dr. Molewaterplein 40

NL-3015 GD Rotterdam, The Netherlands 